\title{
Persistence and Transcription of Paternal mtDNA Dependent on the Delivery Strategy Rather than Mitochondria Source in Fish Embryos
}

\author{
Liangyue Peng ${ }^{a, b} \quad$ Ming Wen ${ }^{a, b} \quad$ Qizhi Liuc Jingyi Peng ${ }^{a, b} \quad$ Sibei Tang ${ }^{a, b}$ \\ Yunhan Hong ${ }^{a}$ Shaojun Liua,b Yamei Xiao ${ }^{a, b}$ \\ aState Key Laboratory of Developmental Biology of Freshwater Fish, Hunan Normal University, \\ Changsha, 'b School of Life Sciences, Hunan Normal University, Changsha, Hunan, China, 'Department of \\ Biological Sciences, National University of Singapore, Singapore, Singapore
}

\section{Key Words}

Heteroplasmy • Mitochondria microinjection - MtDNA • Maternal inheritance - Transcriptional quiescence

\begin{abstract}
Background/Aims: Mitochondria (MT) and mitochondrial DNA (mtDNA) show maternal inheritance in most eukaryotic organisms; the sperm mtDNA is usually delivered to the egg during fertilization and then rapidly eliminated to avoid heteroplasmy, which can affect embryogenesis. In our previous study, fertilization-delivered sperm mtDNA exhibited late elimination and transcriptional quiescence in cyprinid fish embryos. However, the mechanisms underlying elimination and transcriptional quiescence of paternal mtDNA are unclear. Methods: Goldfish and zebrafish were used to investigate the fate of mtDNAs with different parental origins delivered by fertilization or microinjection in embryos. Goldfish MT from heart, liver and spermatozoa were microinjected into zebrafish zygotes, respectively. Specific PCR primers were designed so that the amplicons have different sizes to characterize goldfish and zebrafish cytb genes or their cDNAs. Results: The MT injection-delivered paternal mtDNA from sperm, as well as those from the heart and liver, was capable of persistence and transcription until birth, in contrast to the disappearance and transcriptional quiescence at the heartbeat stage of fertilization-delivered sperm mtDNA. In addition, the exogenous MT-injected zebrafish embryos have normal morphology during embryonic development. Conclusions: The fate of paternal mtDNA in fishes is dependent on the delivery strategy rather than the MT source, suggesting that the presence of sperm factor(s) is responsible for elimination and transcriptional quiescence of fertilization-delivered sperm mtDNA. These findings provide insights into the mechanisms underlying paternal mtDNA fate and heteroplasmy in cyprinid fishes.

L. Peng, M. wen and Q. Liu contributed equally to this work

Yamei Xiao




\section{Cellular Physiology Cell Physiol Biochem 2018;47:1898-1908 \\ \begin{tabular}{c|c|c|} 
DOI: 10.1159/000491070 & O 2018 The Author(s). Published by S. Karger AG, Basel \\
www.karger.com/cpb
\end{tabular} \\ Peng et al.: Persistence and Expression of Paternal mtDNA in Fish}

\section{Introduction}

The mitochondrial genome is believed to show maternal uniparental inheritance (MUI) in many eukaryotes. Sperm-derived paternal mitochondria (MT) normally enter the oocyte cytoplasm upon fertilization and then disappear during early embryogenesis. In a diverse range of animals, there are different mechanisms underlying MUI of mitochondrial DNA (mtDNA) through the exclusion or elimination of paternal mtDNA[1-3]. These mechanisms fall into two major categories: pre-fertilization and post-fertilization mechanisms. Prefertilization mechanisms primarily operate in Drosophila, in which two strategies are used to degrade mtDNA by mitochondrial endonuclease $G$ during spermatid elongation and to extrude mtDNA during sperm individualization, leading to the exclusion or elimination of paternal mtDNA[4, 5]_ENREF_4. Post-fertilization mechanisms operate in Caenorhabditis elegans, where sperm MTs are ubiquitinated and undergo proteasomal mitophagy, leading to pre-blastocyst/blastula elimination of sperm mtDNA until the 16-64-cell stages [6]. Both pre- and post-fertilization mechanisms are utilized to avoid paternal mtDNA inheritance in vertebrates such as pig [7] and medaka [8].

Many organisms possess a pool of homogeneous mtDNA molecules. This mitochondrial homoplasmy is essential for normal development, as heteroplasmy comprising different wildtype mtDNAs may cause genetic instability, cognitive or behavioral impairment in mouse [9] and diseases in humans [10]. The mechanism underlying homoplasmy is uniparental inheritance of mtDNA. In particular, MUI of mtDNA prevails in numerous organisms, including many invertebrates and all vertebrate species, such as humans and other mammals $[11,12]$. Recent studies have revealed that paternal inheritance or leakage of mtDNA may occur to variable degrees even in organisms with demonstrated MUI, such as Drosophila $[13,14]$. In humans, paternal inheritance of mtDNA is controversial. On one hand, linkage disequilibrium and recombination in mtDNA suggest paternal inheritance of mtDNA[15], and a patient carrying a pathogenic mtDNA mutation has been considered the best evidence for paternal inheritance of human mtDNA[16]. On the other hand, subsequent analyses of patients carrying various mtDNA defects have argued against paternal inheritance of human $\operatorname{mtDNA}[17,18]$.

In fish, MUI has been reported in medaka [8]. Most recently, we observed MUI in cyprinid fish, e.g., goldfish and blunt-snout bream, in which paternal mtDNA was present in zygotes but absent in larvae and adult organs. Meanwhile, we also found that the cytb RNA of paternal mtDNA was not present in all stages, while paternal mtDNA was easily detectable, and revealed that fertilization-delivered paternal mtDNA is transcriptionally quiescent and thus does not lead to the development of heteroplasmic embryos [19]. However, the cause of paternal mtDNA elimination and transcriptional quiescence remains unclear.

To further elucidation of this issue, in this study, goldfish (Carassius auratus red var.) and zebrafish (Danio rerio) were used as experimental animals in this study to analyze the possible effects of MT source and delivery strategy on the fate of paternal mtDNA. Our results demonstrated that MT injection-delivered mtDNA from not only the somatic organs heart and liver but also sperm is capable of persistence and transcription throughout embryogenesis. These results demonstrate that the fate of paternal mtDNA is dependent on the delivery strategy rather than the MT source. In addition, the normal morphology of exogenous MT-injected zebrafish embryos also indicates that mitochondrial heterogeneity in cyprinid species does not cause abnormal embryonic development.

\section{Materials and Methods}

\section{Ethics statement}

Animal experimenters are licensed after attending a training course on laboratory animals held by the Institute of Experimental Animals, Hunan Province, China. Procedures were conducted following the regulations of the Administration of Affairs Concerning Experimental Animals for the Science and Technology Bureau of China. 


\section{Cellular Physiology Cell Physiol Biochem 2018;47:1898-1908 \begin{tabular}{ll|l} 
and Biochemistry Published online: June 29, 2018 & $\begin{array}{l}\text { (C) } 2018 \text { The Author(s). Published by S. Karger AG, Basel } \\
\text { www.karger.com/cpb }\end{array}$
\end{tabular}}

\section{Fish}

Fish work was performed in strict accordance with the recommendations in the Guidelines for the Care and Use of Laboratory Animals of the National Advisory Committee for Laboratory Animal Research in China and was approved by the Animal Care Committee of Hunan Normal University (Permit Number: 4237). Goldfish (red variety; C. auratus) was maintained at the Ministry of Education National Center of Polyploidy Fish Breeding, Hunan Normal University as described previously [20, 21]. Intraspecific mating and interspecies hybridization were conducted using the method of dry artificial insemination. Embryos were placed on nylon meshes in water for mass production or in Petri dishes for experimentation. Embryos in Petri dishes were regularly monitored, snap-frozen in liquid nitrogen at different stages and stored at $-80^{\circ} \mathrm{C}$ before use. Zebrafish (D. rerio) was maintained at the State Key Laboratory of Developmental Biology of Freshwater Fish, College of Life Sciences, Hunan Normal University as described previously [22].

\section{Mitochondrial preparation and activity}

MTs were isolated from approximately $100 \mathrm{mg}$ of adult organs (heart, muscle and liver) and semen of goldfish and zebrafish using a Mitochondrial Isolation Kit (Sciencell Cat. No. 8286) and suspended in $100 \mu \mathrm{l}$ of buffer B for storage at $-80^{\circ} \mathrm{C}$ until use. Protein concentration was measured using a BSA protein quantification kit (Auragne, Cat. No. P001B-1). Mitochondrial DNA was released from MT aliquots by heating for $15 \mathrm{~min}$ at $95^{\circ} \mathrm{C}$ on a thermal cycler (Applied Biosystems) and measured for optical density on a Synergy-2 microplate reader (BioTek, Winooski). Mitochondrial preparations were diluted with buffer $\mathrm{B}$ to a concentration of $0.1 \mu \mathrm{g} / \mathrm{ml}$ protein. Cytochrome oxidase activity was measured using the GENMED Cytochrome Oxidase Measurement Kit (GMS10014.2.v.A).

\section{Mitochondrial microinjection}

Approximately $1 \mathrm{nl}$ of Buffer B or mitochondrial preparation at $0.001 \sim 0.1 \mu \mathrm{g} / \mu \mathrm{l}$ protein was injected into embryos at the 1-cell stage (post-fertilization in 5-10 minutes) using a FemtoJet (Eppendorf, Germany). These dosages were equivalent to 100 10, 000 mtDNA copies per injection. Microinjected embryos were reared in Petri dishes in water at $22^{\circ} \mathrm{C}$ (goldfish) or in zebrafish egg water at $28^{\circ} \mathrm{C}$ (zebrafish). Zebrafish embryos were microinjected at the 1-cell stage (5-10 minutes post-fertilization) with MT from organs of heart, liver and sperm and examined for survival and phenotype at critical stages.

\section{DNA and RNA extraction}

Before DNA and RNA extraction of the embryos, the zona pellucida and spermatozoa attached to or within the perivitelline space were removed by washing with acidified Tyrode's solution. DNA was extracted from freshly dissected organs or groups $(n>20)$ of frozen embryos using the TaKaRa Mini BEST Universal Genomic DNA Extraction Kit (TaKaRa, Japan) as described previously [20]. RNA was extracted using the E.Z.N.A. Total RNA Kit II (OMEGA).

\section{Plasmid}

Plasmid pMD18 cytb was constructed as a reference to determine the copy number of mtDNA by PCR analysis. The 979-bp fragment of the goldfish cytb was PCR-amplified and cloned into pMD18-T (2692 bp).

\section{Polymerase chain reaction}

Genomic DNA PCR was run for 35 cycles $\left(94^{\circ} \mathrm{C}\right.$ for 30 s, $58^{\circ} \mathrm{C}$ for 30 s and $72^{\circ} \mathrm{C}$ for 30 s) in a $25 \mu \mathrm{l}$ volume containing 50ng of template DNA and appropriate primers for $c y t b$ and $\beta$-actin as described previously [19]. Goldfish DNA, zebrafish DNA or a mixture with serial dilutions were used as template DNA samples. For RT-PCR, firststrand cDNA was synthesized using the PrimeScript ${ }^{\mathrm{TM}}$ RT reagent Kit with gDNA Eraser

Table 1. Primers used for PCR analysis

\begin{tabular}{|c|c|c|c|c|}
\hline \multirow{2}{*}{ Gene } & \multicolumn{2}{|r|}{ Primers } & \multicolumn{2}{|c|}{ PCR product (bp) } \\
\hline & Species (accession) & Name: sequence & & CDNA \\
\hline \multirow{3}{*}{ cytb } & goldfish (JN105355) & $\begin{array}{l}\text { cytbG: GTTGACCTACCCACACCATCC } \\
\text { cytbR: TTTCTACTCATCCTGCTAGTGG }\end{array}$ & 979 & 979 \\
\hline & zebrafish (NC_002333) & $\begin{array}{l}\text { cytbZ: GAAACATCGGAGTAGTCCTGTTCT } \\
\text { cytbR: TTTCTACTCATCCTGCTAGTGG }\end{array}$ & 696 & 696 \\
\hline & goldfish \&zebrafish & $\begin{array}{l}\text { cytbF: CGAGATGTAAACTACGGCTGAC } \\
\text { cytbR:TTTCTACTCATCCTGCTAGTGG }\end{array}$ & 823 & 823 \\
\hline \multirow{3}{*}{ tfam } & goldfish (KT380497) & $\begin{array}{l}\text { tfamG: GTCGGTGCGAATCTTCTGACG } \\
\text { tfamR: GAGATCGGAAGAGAAGACCTT }\end{array}$ & 659 & 659 \\
\hline & zebrafish (NM_001077389) & $\begin{array}{l}\text { tfamZ: GCAGTGGAAAATGTTGACCACCG } \\
\text { tfamR: GAGATCGGAAGAGAAGACCTT }\end{array}$ & 433 & 433 \\
\hline & goldfish \&zebrafish & $\begin{array}{l}\text { tfamF: GTTGTGAGGTGTTCATGTGTAG } \\
\text { tfamR: GAGATCGGAAGAGAAGACCTT }\end{array}$ & 607 & 607 \\
\hline
\end{tabular}


(TaKaRa), and PCR was run for 35 cycles in a $25 \mu \mathrm{l}$ volume containing $10 \mathrm{ng}$ of template cDNA and appropriate primers for $c y t b$ or 30 cycles for $\beta$-actin as a loading control. The primers used are listed in Table 1. PCR products were separated on $1.5 \%$ agarose gels and observed on White/UV Transilluminators (UVP, Upland, CA 91786).

\section{Sequence analysis}

Sequences were analyzed using BLAST search and aligned with Vector NT.

\section{Microscopy}

Observation and photography were performed under a Leica MZFIII stereo microscope, a Zeiss Axiovert inverted and a Axiovert upright microscopes with a Zeiss AxioCam M5Rc digital camera (Zeiss Corp) as described previously [19].

\section{Statistics}

Statistical analyses were calculated using GraphPad Prism v4.0. Data are presented as mean \pm s.d., and $p$ values were calculated using non-parametric Student's $t$-test.

\section{Results}

Hybrid embryos between goldfish and zebrafish are an ideal model system to study the mechanism of MUI

Because zebrafish embryos can be easily obtained and microinjected, we used goldfish and zebrafish as experimental animals to further investigate the fate of mtDNAs with different parental origins delivered by fertilization or microinjection in embryos (Fig. 1a and b). A pair-wise comparison revealed that goldfish and zebrafish shared 78.1\% identical mtDNA sequences. Specifically, sequence alignment allowed for designing PCR primers that were common or specific to mtDNAs of distinct parental origins, and there was a 78\% sequence identity of $c y t b$ as a representative mtDNA gene (Fig. 2) and a 69.9\% sequence identity of tfam as a representative nuclear gene (Fig. 3). The PCR primers were designed so that the amplicons with different sizes characterize of goldfish and zebrafish cytb genes or their transcripts (Fig. 1c and d). By using a cytb-bearing plasmid as reference, we were able to measure the absolute copy number of mtDNA

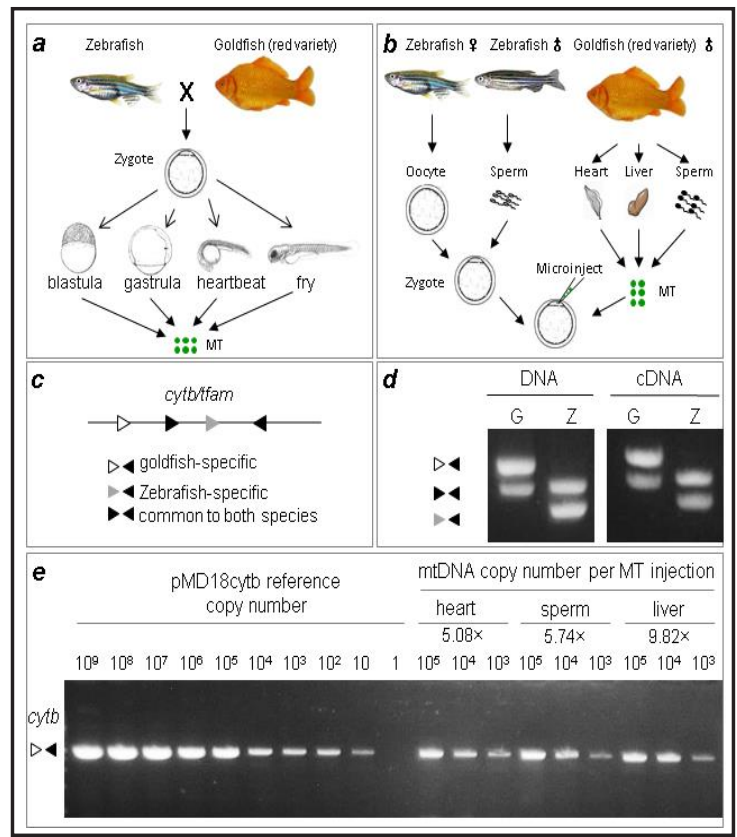

Fig. 1. Fishes and detect strategy. (a) Flow chart of the goldfish red variety and zebrafish used for hybridization. (b) Flow chart of goldfish mitochondrial injection into zebrafish. (c) Scheme of PCR primers for cytb and tfam, showing primers specific to goldfish (open arrowhead) or zebrafish (gray arrowhead) and common to both species (black arrowheads). (d) Detection of DNA and cDNA by specific primers. Z, zebrafish; G, goldfish. (e) Determination of mtDNA copy number in microinjected goldfish MT. Three ng of pMD18cytb corresponding to $10^{9}$ plasmid copies was serially diluted and used as a copy number standard for comparison by PCR. MT were injected at $0.1,0.01$ and $0.001 \mu \mathrm{g} /$ of protein containing estimated copy numbers (see also Table 1).

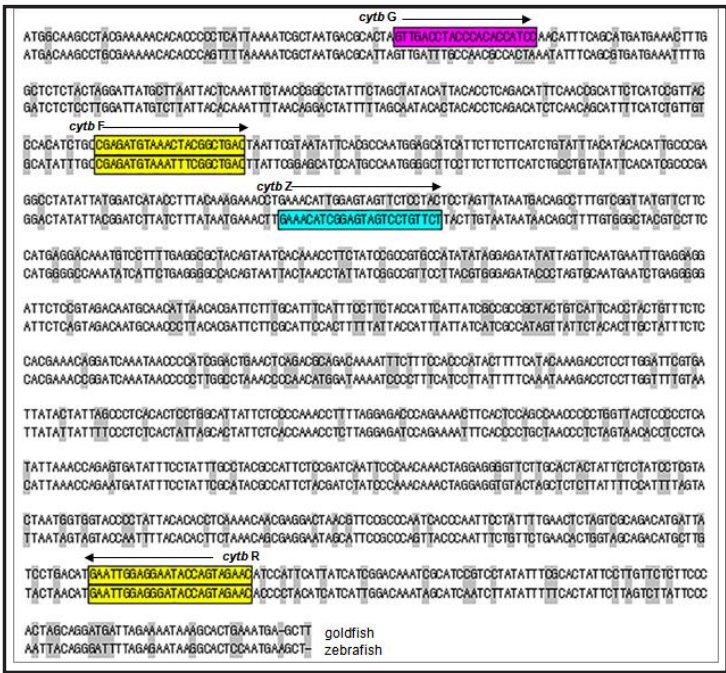

Fig. 2. Alignment of cytb nucleotide sequences. 
in MT preparations for microinjection by PCR analysis (Fig. 1e). Therefore, forward and backcross hybrids between zebrafish and goldfish can provide a suitable model system to quantify the mtDNAs of different parental origins by sensitive PCR assays.

The fate of fertilization-delivered sperm mtDNA in goldfish and zebrafish hybrids Using a cytb-bearing plasmid as a reference, we tested MUI in the forward and backcross hybrids between goldfish and zebrafish. Regardless of whether the female parent was a goldfish or a zebrafish , only maternal mtDNA could be detected in the hybrids, whereas paternal mtDNA was absent (Fig. 1d), suggesting strict MUI of mtDNA in goldfish and zebrafish hybrids.

To examine the fate of paternal mtDNA during embryogenesis, we used embryos from forward and backcross hybrids for genomic DNA isolation and analyzed the samples by PCR at representative stages indicated. As expected, the egg mtDNA was evident throughout embryogenesis. However, sperm mtDNA was still easily detected at the blastula, gastrula and even heartbeat stages but disappeared before hatching (Fig. 4a; lanes 1-2, 6 and 10). The delayed elimination of paternal mtDNA described above prompted us to examine the transcriptional status of maternal and paternal mtDNA at critical stages of development. The cytb transcript of paternal origin was never detected in forward and backcross hybrid embryos at any of the stages examined (Fig. 4b).

Fig. 4. Late elimination and transcriptional quiescence of paternal mtDNA. (a) cytb expression in the embryos of goldfish (G) and zebrafish (B). Persistence of sperm mtDNA in developing embryos was observed until the heartbeat stage $(24 \mathrm{~h}$ post-fertilization) and its disappearance in fry around hatching. (b) Lack of cytb expression from paternal mtDNA of zebrafish (asterisks) and goldfish (hashes) in hybrid embryos. Embryos from parental species and hybrids were analyzed by RT-PCR at the indicate representative stages. $\beta$-actin was used as a loading control. PCR and gels were run under the same conditions. For abbreviations, see legend to Fig. 1.

\begin{abstract}
ATCGCTOCATTCAGCTTGATGTCAGTGGGGGCIAATCTCCTGGTCAAGTCATTCAGC-CT ATCGCTOCGTTCGCCTTGATGTCA GTOGGTGCGAATCTTCTGACG HAGTO-TTTCGCTCT tfam $\mathrm{G}$

GTTCTCGAGTCCATCT GTTGTGAGGTGTTCATGTGTAGCTCCAGCGATAAAGAGTTTTAG GTTCTCGAGCOCATCTETTGIGAGGTGITCATGTGTACCTCCAGTGTTAAAGAGTTTAG $\operatorname{tam} \mathrm{F}$

CACTGCAACTOGAGGTCCACCCAAGAGACCOCTGACAGCATACATCACATTTGTAAAGGA CAOCACAACOCGGCGTCCACCCAAGAGACCOCTGACAGCATACATTACATATGTGAAAGA

TATGCAGCCCACOGTCAGCAAACAAAATCOTAGCA-TAAAAGTGTCGATGTTATCCGAA GATGCATCCCAACATCACCAGACAAATCO-AGGAGTCAAHATGTCGATATTGTOCGAA tram Z

AGATTCOCCA ECAGTGGAAAATGTTGACCACOCA iACAAAAGCAGCCATTTCAGGTTCOCT AGTTAGCACACCAGTGGAAGTCCTGACTCOTGAACAGAAGCAGCCATTTCAGGATGCAT

CTCTGGAGGOCAAGGAGCAATATAAACTTGOCTTGGAGAAATTCAAAGCOCAGCTCACOC CITTGCCATCAAAGGAGCAATATAAGCTTGAGGTGGAGAAATACAAACCCCAACTGAGTC

CTGCAGAGAGTGOCGCCITTTGCOGAGGAAAACGACAAAGAGTGCCCAAAAGAAAGCCAA CTCCACAGACTGOTCCTCTTGCGATAGAAAAACGTCAAAAAGTCGCCAAAOCAAAACCAA

TTOGGAAAAAAGGAATTGAATAATCTTGGCAAGCCCAAACGCCCÄAGGAGCAOCTTCA TCAGGAGGAAGAAGGAGTTGAACAGTCTTGGCAAGCCCAAACGCCOCAGGAGCGOCTTCA

ACATCTTCATCGCGGAGCATTTTGTGGAGGCAAAGGGAACTACTACGCAGCCAAAATTGA ACATCTTCATGTCGGAGCACTTTGAGGAAGCAAAGGAAATAATATGCAGACAAAGATGA

AGTCACTAAGAGATGACTGGAATOGCTTGAGCGACACACAAAAACAAATGTACATACAGC AGTCGCTAAGAGACGACTGGGAGOGITTCAGTGCAACACAGAACAAATGTACATTCAGC

TGCCAGAGGAOGATAAAGTCCGTTACAAGAATGAATCAAATOGTGCGAGGAGCACATGA TGGCTGAGGATGACAAAGTCCGTTACAAGACGAAATAAATOGTGCGAGGAACACATGA $\longleftarrow$ tram R

TCEAGATCCGAAGAGAAGACCTICTGOGGAGAAH-GACGAAGAGCGCO zebrafish TA GAGATCGGAAGCGAAGACTIGTTOCGAGAAAAGA-GAGGAGACOC goldfish
\end{abstract}

Fig. 3. Alignment of tfam nucleotide sequences.
Table 2. Yield, mtDNA copy and activity of MT from different organs. ${ }^{1)}$ Calculated on the basis of 16,580 bp known for the goldfish mtDNA (gene accession number, GI:56798128). ${ }^{2)} \mathrm{Cy}-$ tochrome $\mathrm{C}$ oxidase activity

\begin{tabular}{|c|c|c|c|c|c|}
\hline MT sample & & Heart & Muscle & Liver & Sperm \\
\hline \multirow{9}{*}{ MT stock preparation } & Wet sample $(\mathrm{g})$ & 0.11 & 0.13 & 0.09 & 0.12 \\
\hline & Volume of MT preparation $(\mu \mathrm{l})$ & 100 & 100 & 100 & 100 \\
\hline & $\mu \mathrm{g}$ MT protein/ $\mu \mathrm{l}$ MT preparation & 4.98 & 1.75 & 5.65 & 9.41 \\
\hline & 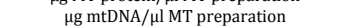 & 0.46 & 0.07 & 0.59 & 1.68 \\
\hline & mtDNA copies/ $/ \mu \mathrm{l}$ MT preparation $\left(10^{9}\right)^{1)}$ & 25.30 & 3.85 & 32.45 & 92.40 \\
\hline & mtDNA copies/ $\mu \mathrm{g}$ MT protein $\left(10^{9}\right)^{1)}$ & 5.08 & 2.20 & 5.74 & 9.82 \\
\hline & $\mu \mathrm{g}$ MT protein/g sample & 45.24 & 13.43 & 62.75 & 78.40 \\
\hline & mtDNA copies/g sample $\left(10^{9}\right)$ & 253.00 & 38.50 & 324.50 & 924.00 \\
\hline & Enzyme activity $(\mu \mathrm{M} / \mu \mathrm{g} / \mathrm{min})^{2)}$ & 83.30 & 28.40 & 9.80 & 14.00 \\
\hline \multirow{3}{*}{ MT working solution } & $\mu \mathrm{g}$ MT protein/ $\mu \mathrm{l}$ MT working solution & 0.10 & 0.10 & 0.10 & 0.10 \\
\hline & mtDNA copies/pl MT working solution $\left(10^{8}\right)$ & 5.08 & 2.20 & 5.74 & 9.82 \\
\hline & mtDNA copies per microinjection $\left(10^{5}\right)$ & 5.08 & 2.20 & 5.74 & 9.82 \\
\hline
\end{tabular}

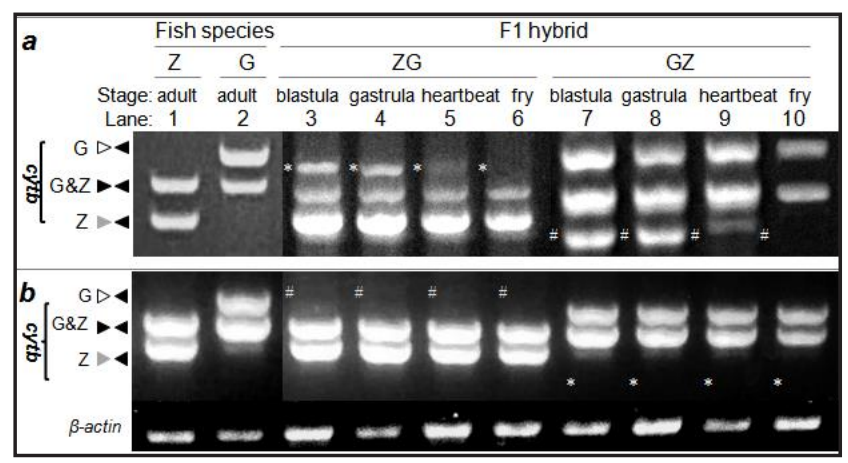




\section{Cellular Physiology Cell Physiol Biochem 2018;47:1898-1908 \begin{tabular}{ll|l} 
and Biochemistry Published online: June 29, 2018 & $\begin{array}{l}\text { (C) } 2018 \text { The Author(s). Published by S. Karger AG, Basel } \\
\text { www.karger.com/cpb }\end{array}$
\end{tabular}

\section{The fate of injection-delivered mtDNAs from several different tissues}

MTs were isolated from the sperm, heart, muscle and liver of goldfish to determine the yield, activity and copy number of mtDNA (Table 2). Sperm gave rise to the highest MT yield $\left(78.4 \mu \mathrm{g}\right.$ of MT protein per gram of sperm) and highest mtDNA copy number $\left(9.82 \times 10^{9}\right.$ copies per $\mu \mathrm{g}$ of MT protein), whereas muscle had the lowest yield $(13.43 \mu \mathrm{g}$ of MT protein per gram of sperm) and the lowest mtDNA copy number $\left(2.2 \times 10^{9}\right.$ copies per $\mu$ g of MT protein). These findings suggested that there are no pre-fertilization mechanisms for sperm mtDNA elimination in fish. Due to the high cytochrome $\mathrm{C}$ oxidase activity of heart MT, we chose heart MT, in addition to MT from liver and sperm, for microinjection (liver and sperm MT microinjection has been reported in mice [23]).

In our pilot experiments, heart MTs from goldfish and zebrafish were microinjected into the zygotes of zebrafish and goldfish, respectively. The microinjected heterogenous heart mtDNA was easily detected in not only the embryos from blastula to gastrula and heartbeat but also in their fry (Fig. 5a). Concurrently, cytb transcripts from the microinjected heart mtDNA were also easily detectable at all of these stages (Fig. 5b), demonstrating the active transcription of MT injection-delivered heterogenous mtDNA from the heart. Next, liver MT from goldfish was microinjected at three different dosages into zebrafish zygotes, and its mtDNA was similarly examined. The goldfish liver mtDNA and its RNA were also observed in experimental zebrafish from birth (Fig. 6a, b). Thus, both heart and liver mtDNA upon delivery by MT injection is capable of persistence and transcription until birth. Lastly, goldfish sperm MT were microinjected at three different dosages into zebrafish zygotes, and

Fig. 5. Persistence and expression of microinjected heart mtDNA. Goldfish (G) and zebrafish (Z) embryos were microinjected at the 1 -cell stage with heart MT $\left(5.08 \times 10^{5}\right.$ mtDNA copies per microinjection, for more details on the embryo number of injected and the embryo number of survived in four respective stages, see Table 2.) from zebrafish or goldfish and analyzed at 4 representative stages for the cytb DNA and cytb RNA. (a) Genomic DNA PCR analysis. (b) RT-PCR analysis. The cytb DNA (asterisks) and cytb RNA (arrowheads) from injected MT were observed throughout embryogen-

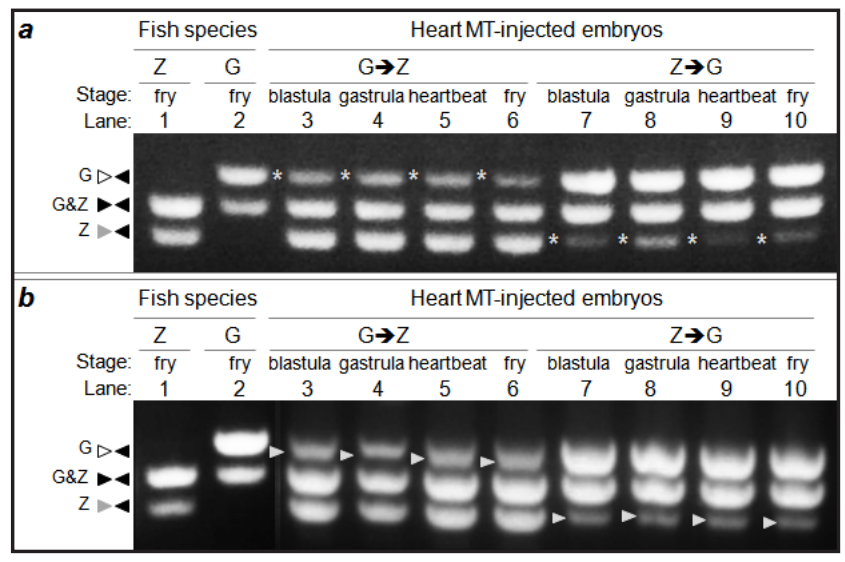
esis. $G \rightarrow Z$, goldfish heart mitochondrial injection into zebrafish; $Z \rightarrow G$, zebrafish heart mitochondrial injection into goldfish.

Fig. 6. Persistence and expression of microinjected liver mtDNA. Zebrafish embryos were microinjected at the 1-cell stage with goldfish liver MT (MT were injected at $0.1,0.01$ and $0.001 \mu \mathrm{g} /$ of protein containing estimated copy numbers $5.74 \times 10^{5}$, $5.74 \times 10^{4}$ and $5.74 \times 10^{3}$, for more details on the embryo number of injected and survived at four representative stages, see Table 2.) and analyzed for the cytb DNA and cytb RNA at 4 representative stages [blastula (bla), gastrula (gas), heartbeat (heart)

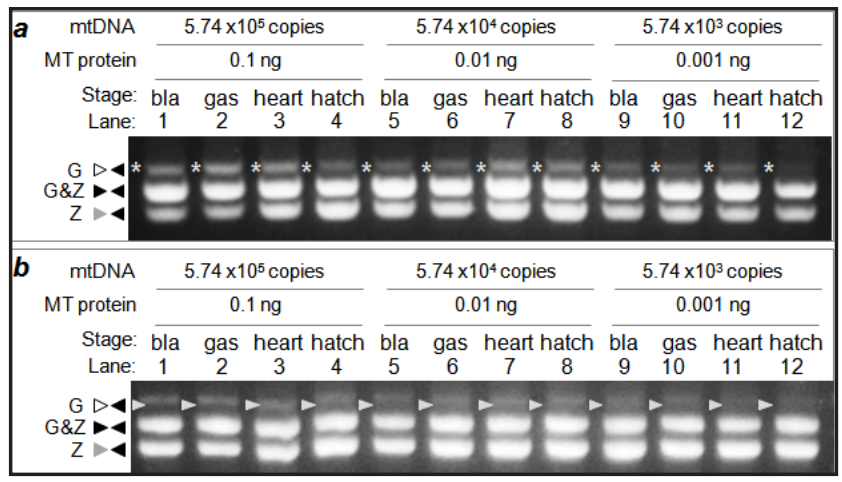
and hatching (hatch)]. (a) Genomic DNA PCR analysis. (b) RT-PCR analysis. The cytb DNA (asterisks) and cytb RNA (arrowheads) from injected liver MT were observed throughout embryogenesis. 
the dynamics of its mtDNA was also monitored. The goldfish sperm mtDNA and its RNA were easily detected throughout zebrafish embryogenesis (Fig. 7a, b). These results showed that sperm mtDNA delivered by MT injection behaves differently from those delivered by fertilization, but resembles heart and liver mtDNA in persistence and transcription.

For comparison, the tfam transcripts of both maternal and paternal origins were readily detected in zebrafish embryos. Zebrafish embryos were microinjected at the 1-cell stage with goldfish liver or sperm MT and analyzed for the tfam RNA at 4 representative stages (blastula, gastrula, heartbeat and hatching). The expression of tfam from injected liver and sperm MT was analyzed by RT-PCR. No goldfish tfam transcripts from injected goldfish liver and sperm MT was detected throughot embryogenesis (Fig. 8a-c).

\section{Heteroplasmy does not have impact on embryogenesis}

The results showing that paternal mtDNA could persist and be transcribed until birth demonstrated the heteroplasmy throughout embryogenesis in cyprinid species. To determine the impact of injection-delivered MT on embryogenesis, we further examined the survival and phenotype at critical stages of zebrafish embryos microinjected with MT from the

Fig. 7. Persistence and expression of microinjected sperm mtDNA. Zebrafish embryos were microinjected at the 1-cell stage with goldfish sperm MT(MT were injected at 0.1, 0.01 and $0.001 \mu \mathrm{g} /$ of protein containing estimated copy numbers $9.82 \times 10^{5}, 9.82 \times 10^{4}$ and $9.82 \times 10^{3}$, for more details on the embryo number of injected and survived at four representative stages, see Table 2.) and analyzed for the cytb DNA and cytb RNA at 4 representative stages [blastula (bla), gastrula (gas), heartbeat (heart) and hatching

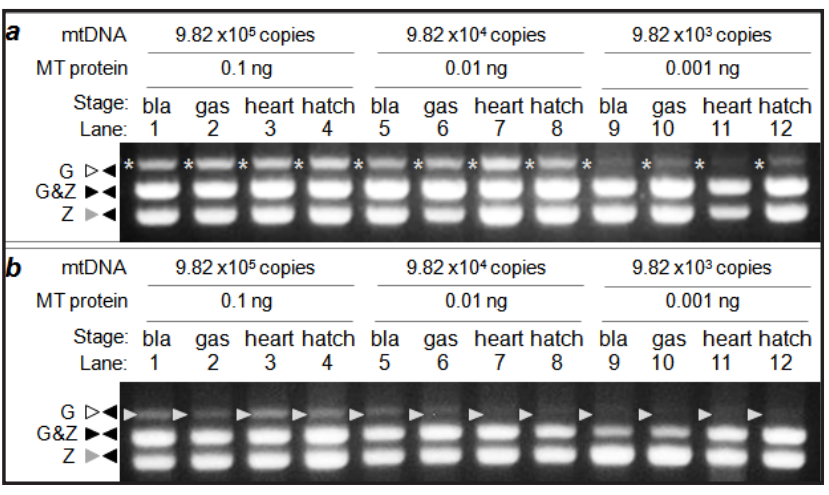
(hatch)]. (a) Genomic DNA PCR analysis. (b)

RT-PCR analysis. The cytb DNA (asterisks) and cytb RNA (arrowheads) from injected sperm MT were observed throughout embryogenesis.

Fig. 8. RT-PCR analysis of tfam from injected liver and sperm MT throughout embryogenesis. Zebrafish embryos were microinjected at the 1-cell stage with goldfish liver and sperm MT (MT were injected at $0.1,0.01$ and $0.001 \mu \mathrm{g} /$ of protein containing estimated copy numbers $5.74 \times 10^{5}$, $5.74 \times 10^{4}$ and $5.74 \times 10^{3}$ in liver and $9.82 \times 10^{5}, 9.82 \times 10^{4}$ and $9.82 \times 10^{3}$ in sperm, for more details on the embryo number of injected and survived at four representative stages, see Table 2.) and analyzed for the tfam RNA at 4 representative stages [blastula (bla), gastrula (gas), heartbeat (heart) and hatching (hatch)]. (a) RT-PCR analysis of tfam from injected liver MT. (b) RT-PCR analysis of tfam from injected sperm MT. (c) RT-PCR analysis of tfam from

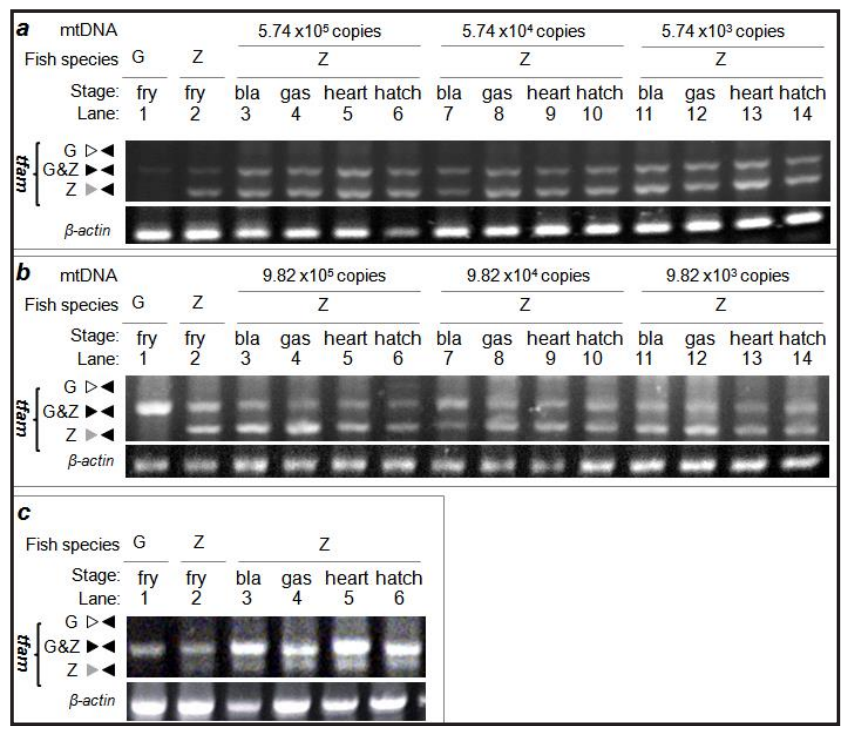
zebrafish throughout embryogenesis. No goldfish tfam RNA from injected goldfish liver and sperm MT was observed throughout embryogenesis. 
heart, liver or sperm at the 1-cell stage. Non-injected control embryos had a $60 \%$ survival rate at hatching and $95 \%$ appareantlyr normal fry, whereas buffer-injected embryos exhibited $\quad 40 \%$ survival and $\quad 90 \%$ appareantly normal fry, indicating that microinjection caused mechanical damages to embryos and slightly compromised their survival and development. Importantly, microinjection of MT from heart, liver or sperm achieved similar survival rates and appareantly normal fry rates compared to buffer injection (Table 3). Phenotypically, MT-injected zebrafish embryos were not different from buffer-injected controls at critical stages, such as heartbeat (Fig. 9a-d) and hatching (Fig. 9a'-d'). Therefore, MT injectionintroduced mtDNA heteroplasmy has little adverse effects on embryonic survival and development of cyprinid fishes.

\section{Discussion}

Mitochondrial DNA is believed to be maternally inherited in many eukaryotes. Sperm-derived paternal MT normally enters the oocyte cytoplasm upon fertilization and then disappears during early embryogenesis. We recently revealed that fertilization-delivered sperm mtDNA from blunt-snoutbream into goldfish eggs was present during early embryogenesis but showed transcriptional quiescence [19], which suggests that parental mtDNA is either intrinsically incapable of transcription or prevented from transcription upon fertilization-mediated delivery in cyprinid embryos. In this study, we demonstrated that parental mtDNA possesses the intrinsic ability for transcription, but this process is somehow inhibited because MT injection-delivered mtDNA from not only heart and liver but also sperm is capable of active transcription throughout cyprinid embryogenesis.

The mechanism of paternal MTs and mtDNA elimination from fertilized oocytes is now partially deciphered. Distinct molecular mechanisms to prevent inheritance of paternal mtDNA have been described in various species. The pre-fertilization mechanism is proposed in Chinook salmon_ENREF_26, where sperms have fewer mtDNA copies for delivery at fertilization of the eggs [24]. In Drosophila, mtDNA is degraded during spermatogenesis, and mature sperms lack any mtDNA and do not deliver paternal mtDNA to ensure MUI[5]. $\mathrm{Yu}$ et al. showed that the mitochondrial DNA polymerase promotes abrupt elimination of mitochondrial genomes during spermatogenesis in Drosophila [25]. Upon fertilization, proteasomal and lysosomal pathways are activated in oocytes to eliminate paternal MT and mtDNA $[6,26,27]$. In C. elegans, two groups have shown that paternal MTs are degraded by fertilization-induced autophagy $[26,28]$. A mitochondrial endonuclease G (CPS-6) has been implicated in breakdown of paternal MTs upon fertilization [4]. In addition, Thompson et al. showed that the inner mitochondrial membrane protein prohibitin (PHB) were the ubiquitinated substrates in mammalian [29]. Wei et al. identified the PHB2 as a crucial mitophagy receptor involved in targeting MT for autophagic degradation. PHB2 is required 


\section{Cellular Physiology Cell Physiol Biochem 2018;47:1898-1908 \\ \begin{tabular}{c|c} 
DOI: 10.1159/000491070 & $\begin{array}{l}\text { O 2018 The Author(s). Published by S. Karger AG, Basel } \\
\text { www.karger.com/cpb }\end{array}$
\end{tabular} \\ Peng et al.: Persistence and Expression of Paternal mtDNA in Fish}

for the clearance of paternal MT after embryonic fertilization in C. elegans [30]. In mammals, sperm-derived paternal mitochondria themselves are thought to undergo selective autophagy, which includes mitophagy. Ubiquitination of the sperm mitochondria during spermatogenesis and after-fertilization has been implicated in the targeted degradation of paternal mitochondria $[27,29,31,32]$. However, it is not known whether or not mtDNA degradation could be delayed in mammalian embryos because of the method of delivery of paternal mitochondrial DNA by injection .

The mechanism of selective elimination of parental mitochondrial DNA in fish is only sporadically reported $[8,19,24]$. In medaka fish, the sperm mtDNAs was rapidly digested just after fertilization, and digestion of paternal mtDNA was achieved before total destruction of mitochondrial structures [24]. The results obtained in this study suggest the presence of an embryonic factor or factors responsible for elimination and/or transcriptional quiescence of fertilization-delivered sperm mtDNA but not MT injection-delivered paternal mtDNA prior to birth. The factor(s) may be delivered by sperm at fertilization or derived from eggs but are triggered or activated by sperm-egg interactions during and after fertilization. These factors may directly or indirectly regulate the activity of mitochondrial transcription factor A (Tfam), a key regulator of mtDNA replication and transcription [33, 34]. In mammals, for example, prohibitin controls Tfam and mtDNA copy number [35], and its ubiquitination in sperm [29] correlates with a reduction in Tfam and mtDNA copy number during spermatogenesis [36]. Antelman et al. showed that TFAM may exert a critical role in porcine gametogenesis and preimplantation embryo development in the domestic pig (Sus scrofa) [37]. Identification of the embryonic factor(s) will shed new light on the post-fertilization mechanisms underlying elimination and transcriptional quiescence of paternal mtDNA.

Heteroplasmy arises from the introduction and propagation of foreign MT into a recipient cytoplast [38]. Heteroplasmy of Mitochondrial DNA is associated with reduced fitness in nematodes [39] and mice [9]. Thus, proper removal of MT is essential for organismal health in a wide range of eukaryotic species $[40,41]$. Maternal mtDNA inheritance prevents the occurrence of heteroplasmy between potentially distant mtDNA haplotypes, which may be necessary to avoid the diffusion and transmission of potentially deleterious sperm mtDNA to the progeny. mtDNA heteroplasmy was observed after nuclear transfer in fish [42]. These results suggest that mitochondrial DNA heteroplasmy may not affect embryogenesis in cyprinid fish. In this study, the persistence and transcription of microinjected MT throughout embryogenesis revealed the presence of mtDNA heteroplasmy. Our finding that MTinjected zebrafish embryos are capable of survival and development indicates that mtDNA heteroplasmy has no detectable effect on embryogenesis in cyprinid fishes.

The mechanism underlying the active digestion of paternal MT and mtDNA is still an open question in fish. Eggs likely play an important role in this process. The results from this study and our previous work indicate that cyprinid fish are ideal model organisms for identification and characterization of the embryonic factor(s) involved in the prehatch elimination of paternal MT and mtDNA.

\section{Acknowledgements}

This work was funded by grants from the National Natural Science Foundation of China (31302175, 31772902), the Hunan Provincial Department of Education Research Project (16B162), the National Basic Research Program of China (2012CB722305), and the Hunan Provincial Natural Science and Technology Major Project (2017NK1031). We also thank Professor Zhong Wan and Zhi-Qiang Zeng for reviewing the manuscript.

\section{Disclosure Statement}

The authors declare to have no conflict of interests. 


\section{Cellular Physiology Cell Physiol Biochem 2018;47:1898-1908

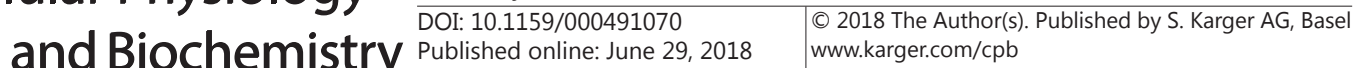 \\ Peng et al.: Persistence and Expression of Paternal mtDNA in Fish}

\section{References}

1 Carelli V: Keeping in shape the dogma of mitochondrial DNA maternal inheritance. PLoS Genet 2015;11:e1005179.

-2 Mishra P, Chan DC: Mitochondrial dynamics and inheritance during cell division, development and disease. Nat Rev Mol Cell Biol 2014;15:634-646.

- Sato M, Sato K: Maternal inheritance of mitochondrial DNA by diverse mechanisms to eliminate paternal mitochondrial DNA. Biochim Biophys Acta 2013;1833:1979-1984.

-4 Zhou Q, Li H, Li H, Nakagawa A, Lin JL, Lee E-S, Harry BL, Skeen-Gaar RR, Suehiro Y, William D: Mitochondrial endonuclease $\mathrm{G}$ mediates breakdown of paternal mitochondria upon fertilization. Science 2016;353:394-399.

5 DeLuca SZ, O'Farrell PH: Barriers to male transmission of mitochondrial DNA in sperm development. Dev Cell 2012;22:660-668.

-6 Sutovsky P, Moreno RD, Ramalho-Santos J, Dominko T, Simerly C, Schatten G: Ubiquitin tag for sperm mitochondria. Nature 1999;402:371-372.

7 Sutovsky P, McCauley TC, Sutovsky M, Day BN: Early degradation of paternal mitochondria in domestic pig (Sus scrofa) is prevented by selective proteasomal inhibitors lactacystin and MG132 Biol Reprod 2003;68:1793-1800.

8 Nishimura Y, Yoshinari T, Naruse K, Yamada T, Sumi K, Mitani H, Higashiyama T, Kuroiwa T: Active digestion of sperm mitochondrial DNA in single living sperm revealed by optical tweezers. Proc Natl Acad Sci U S A 2006;103:1382-1387.

-9 Sharpley MS, Marciniak C, Eckel-Mahan K, McManus M, Crimi M, Waymire K, Lin CS, Masubuchi S, Friend N, Koike M, Chalkia D, MacGregor G, Sassone-Corsi P, Wallace DC: Heteroplasmy of mouse mtDNA is genetically unstable and results in altered behavior and cognition. Cell 2012;151:333-343.

10 Stewart JB, Chinnery PF: The dynamics of mitochondrial DNA heteroplasmy: implications for human health and disease. Nat Rev Genet 2015;16:530-542.

11 Giles RE, Blanc H, Cann HM, Wallace DC: Maternal inheritance of human mitochondrial DNA. Proc Natl Acad Sci U S A 1980;77:6715-6719.

12 Hutchison CA, 3rd, Newbold JE, Potter SS, Edgell MH: Maternal inheritance of mammalian mitochondrial DNA. Nature 1974;251:536-538.

13 Dokianakis E, Ladoukakis ED: Different degree of paternal mtDNA leakage between male and female progeny in interspecific Drosophila crosses. Ecol Evol 2014;4:2633-2641.

14 Wolff JN, Nafisinia M, Sutovsky P, Ballard JW: Paternal transmission of mitochondrial DNA as an integral part of mitochondrial inheritance in metapopulations of Drosophila simulans. Heredity 2013;57-62.

15 Awadalla P, Eyre-Walker A, Smith JM: Linkage disequilibrium and recombination in hominid mitochondrial DNA. Science 1999;286:2524-2525.

16 Schwartz M, Vissing J: Paternal inheritance of mitochondrial DNA. N Engl J Med 2002;347:576-580.

17 Zhang Q, Raoof M, Chen Y, Sumi Y, Sursal T, Junger W, Brohi K, Itagaki K, Hauser CJ: Circulating mitochondrial DAMPs cause inflammatory responses to injury. Nature 2010;464:104-107.

18 Taylor RW, McDonnell MT, Blakely EL, Chinnery PF, Taylor GA, Howell N, Zeviani M, Briem E, Carrara F, Turnbull DM: Genotypes from patients indicate no paternal mitochondrial DNA contribution. Ann Neurol 2003;54:521-524.

19 Wen M, Peng L, Hu X, Zhao Y, Liu S, Hong Y: Transcriptional quiescence of paternal mtDNA in cyprinid fish embryos. Sci Rep 2016;6:28571.

-20 You CP, Zhao RR, Hu J, Liu SJ, Tao M, Zhang C, Chen YB, Qin QB, Xiao J, Duan W, Liu Y: Inheritance of the complete mitochondrial genomes in three different ploidy fishes. Curr Mol Med 2014;14:1322-1330.

21 Hu FZ, Xu K, Zhou YF, Wu C, Wang S, Xiao J, Wen M, Zhao RR, Luo KK, Tao M, Duan W, Liu SJ: Different expression patterns of sperm motility-related genes in testis of diploid and tetraploid cyprinid fish. Biol Reprod 2017;96:907-920.

22 Xiao Y, Zhou Y, Xiong Z, Zou L, Jiang M, Luo Z, Wen S, Liu W, Liu S, Li W: Involvement of JNK in the embryonic development and organogenesis in zebrafish. Mar Biotechnol (NY) 2013;15:716-725.

-23 Shitara H, Kaneda H, Sato A, Inoue K, Ogura A, Yonekawa H, Hayashi JI: Selective and continuous elimination of mitochondria microinjected into mouse eggs from spermatids, but not from liver cells, occurs throughout embryogenesis. Genetics 2000;156:1277-1284. 


\section{Cellular Physiology Cell Physiol Biochem 2018;47:1898-1908 \begin{tabular}{lll} 
DOI: 10.1159/000491070 & $\begin{array}{l}\text { O 2018 The Author(s). Published by S. Karger AG, Basel } \\
\text { www.karger.com/cpb }\end{array}$ \\
\hline
\end{tabular}}

24 Wolff JN, Gemmell NJ: Lost in the zygote: the dilution of paternal mtDNA upon fertilization. Heredity 2008;101:429-434.

25 Yu ZS, O’Farrell PH, Yakubovich N, DeLuca SZ: The Mitochondrial DNA Polymerase Promotes Elimination of Paternal Mitochondrial Genomes. Current Biology 2017;27:1033-1039.

-26 Zhou Q Li H, Xue D: Elimination of paternal mitochondria through the lysosomal degradation pathway in C. elegans. Cell Res 2011;21:1662-1669.

-27 Song WH, Yi YJ, Sutovsky M, Meyers S, Sutovsky P: Autophagy and ubiquitin-proteasome system contribute to sperm mitophagy after mammalian fertilization. Proc Natl Acad Sci U S A 2016;113:E5261-5270.

-28 Sato M, Sato K: Degradation of Paternal Mitochondria by Fertilization-Triggered Autophagy in C. elegans Embryos. Science 2011;334:1141-1144.

-29 Thompson WE, Ramalho-Santos J, Sutovsky P: Ubiquitination of prohibitin in mammalian sperm mitochondria: possible roles in the regulation of mitochondrial inheritance and sperm quality control. Biol Reprod 2003;69:254-260.

30 Wei YJ, Chiang WC, Sumpter R, Mishra P, Levine B: Prohibitin 2 Is an Inner Mitochondrial Membrane Mitophagy Receptor. Cell 2017;168:224-238.

-31 Sutovsky P, Moreno RD, Ramalho-Santos J, Dominko T, Simerly C, Schatten G: Ubiquitinated sperm mitochondria, selective proteolysis, and the regulation of mitochondrial inheritance in mammalian embryos. Biol Reprod 2000;63:582-590.

-32 Al Rawi S, Louvet-Vallee S, Djeddi A, Sachse M, Culetto E, Hajjar C, Boyd L, Legouis R, Galy V: Postfertilization Autophagy of Sperm Organelles Prevents Paternal Mitochondrial DNA Transmission. Science 2011;334:1144-1147.

33 Lu B, Lee J, Nie X, Li M, Morozov YI, Venkatesh S, Bogenhagen DF, Temiakov D, Suzuki CK: Phosphorylation of human TFAM in mitochondria impairs DNA binding and promotes degradation by the AAA+ Lon protease. Mol Cell 2013;49:121-132.

-34 Litonin D, Sologub M, Shi Y, Savkina M, Anikin M, Falkenberg M, Gustafsson CM, Temiakov D: Human mitochondrial transcription revisited: only TFAM and TFB2M are required for transcription of the mitochondrial genes in vitro. J Biol Chem 2010;285:18129-18133.

-35 Kasashima K, Sumitani M, Satoh M, Endo H: Human prohibitin 1 maintains the organization and stability of the mitochondrial nucleoids. Exp Cell Res 2008;314:988-996.

-36 Rantanen A, Jansson M, Oldfors A, Larsson NG: Downregulation of Tfam and mtDNA copy number during mammalian spermatogenesis. Mamm Genome 2001;12:787-792.

37 Antelman J, Manandhar G, Yi YJ, Li R, Whitworth KM, Sutovsky M, Agca C, Prather RS, Sutovsky P: Expression of mitochondrial transcription factor A (TFAM) during porcine gametogenesis and preimplantation embryo development. J Cell Physiol 2008;217:529-543.

38 Cummins JM: Cytoplasmic inheritance and its implications for animal biotechnology. Theriogenology 2001;55:1381-1399.

-39 Liau WS, Gonzalez-Serricchio AS, Deshommes C, Chin K, LaMunyon CW: A persistent mitochondrial deletion reduces fitness and sperm performance in heteroplasmic populations of C. elegans. BMC Genet 2007;8:8.

40 Green DR, Levine B: To be or not to be? How selective autophagy and cell death govern cell fate. Cell 2014;157:65-75.

-41 Redmann M, Dodson M, Boyer-Guittaut M, Darley-Usmar V, Zhang J: Mitophagy mechanisms and role in human diseases. Int J Biochem Cell Biol 2014;53:127-133.

42 Sun YH, Zhu ZY: Cross-species cloning: influence of cytoplasmic factors on development. J Physiol 2014;592:2375-2379. 